12,18

\title{
Графен/нанотрубные квази-1D-структуры в сильных электрических полях
}

\author{
(C) О.Е. Глухова, М.М. Слепченков
}

Саратовский национальный исследовательский государственный университет им. Н.Г. Чернышевского, Саратов, Россия

E-mail: glukhovaoe@info.sgu.ru

Поступила в Редакцию 26 декабря 2021 г.

В окончательной редакции 21 января 2022 г.

Принята к публикации 21 января 2022 г.

Проведены in silico исследования поведения графен/нанотрубных квази-одномерных структур (1D) с ковалентными связями между графеном и нанотрубкой в сильных электрических полях с напряженностью $10^{7}-10^{8} \mathrm{~V} / \mathrm{cm}$. Исследованы атомное строение, зонная структура, функция пропускания электронов, электропроводность и закономерности изменения электронной структуры в сильных полях. Обнаружено, что в отличие от нанотрубки и графена функция пропускания электронов квази-1D-структур имеет пик интенсивности на уровне Ферми. В результате квантового молекулярно-динамического моделирования установлены закономерности деформации атомного каркаса и его разрушения под действием пондеромоторной силы электрического поля. Установлено критическое значение напряженности, при котором электрическое поле отрывает графен от трубки. Оно составляет $\sim 2 \cdot 10^{8} \mathrm{~V} / \mathrm{cm}$. Дальнейшее увеличение напряженности электрического поля приводит к отрыву графена от трубки с ее одновременным разрушением.

Ключевые слова: графен/нанотрубные структуры, электропроводность, пондеромоторная сила, сильные электрические поля.

DOI: $10.21883 /$ FTT.2022.05.52340.264

\section{1. Введение}

Углеродные нанотрубки (УНТ) и графен привлекают внимание исследователей во всем мире на протяжении последних десятилетий благодаря своим исключительным физическим свойствам, в том числе большой удельной поверхности, высокой электро- и теплопроводности, подвижности носителей, прозрачности, микромеханической прочности и гибкости [1-3]. Объединение УНТ и графена в гибридные структуры открывает пути для получения новых материалов с многообещающими свойствами, обусловленными синергетическим эффектом от сочетания структур разной размерности [4-10]. Среди существующих топологических конфигураций графеннанотрубного гибридного материала можно выделить две большие группы: гибридные структуры с горизонтально ориентированными УНТ [11-16] и с вертикально ориентированными УНТ [17-21]. Гибридные структуры с вертикально ориентированным УНТ, называемые в ряде случаев „колонным графеном“, находят свое применение при создании электродов аккумуляторных батарей [22], элементов фононных устройств [23] и наномеханических сенсоров [24], а также в качестве газоразделительной мембраны [25] и материалов теплового интерфейса [26]. На основе гибридных структур графен/УНТ с горизонтально ориентированными нанотрубками разрабатываются высокочувствительные фотодетекторы [27], датчики влажности [28], газовые сенсоры [29], литий-ионные батареи [30] и суперконденсаторы [31].
Большой интерес представляют исследования, направленные на получение соединений из ковалентносвязанных нанотрубок и графена [17-21,32-34]. Подобные соединения обладают улучшенными электрическими и механическими свойствами по сравнению со свойствами атомарно тонкого графена [32]. Для выявления закономерностей влияния особенностей атомного строения на свойства ковалентно-связанных графен-УНТкомпозитов и прогнозирования потенциальных областей их применения широко используются методы компьютерного моделирования [35-48]. В частности, в ряде работ рассматриваются гетероструктуры УНТ-графен с горизонтально ориентированными одностенным нанотрубками (ОУНТ) типа zigzag $(12,0)$ или $(8,0)$, ковалентносвязанными с одной или несколькими графеновыми нанолентами, ширина которых равна длине ОУНТ [38-40]. В структурах этого типа на границе раздела между трубкой и графеном формируется $s p^{3}$-гибридизованная связь, благодаря которой композит графен-ОУНТ имеет гораздо более высокий модуль Юнга по сравнению с отдельными нанотрубкой и графеном, демонстрирует появление пиков Ван Хова в распределении плотности электронных состояний, а также открытие энергетической щели порядка нескольких сотен $\mathrm{eV}$ при контракте наноленты и нанотрубки с полупроводниковым типом проводимости. Довольно много теоретических работ посвящено моделированию бесшовных гетероструктур УНТ-графен с вертикально ориентированными нанотрубками и изучению их электронных и теплопроводящих свойств [42-48]. В работе [43] были предложе- 
ны различные топологические варианты гетероструктур УНТ-графен на базе трубки $(6,6)$, в том числе конфигурации с нанотрубками, закрытыми или открытыми с концов. С помощью метода сильной связи авторами прогнозируется, что несмотря на металлический характер проводимости УНТ $(6,6)$ и листа графена, гетероструктуры УНТ $(6,6)$-графен характеризуются наличием запрещенной зоны величиной $0.27 \mathrm{eV}$ (случай с открытыми концами УНT) и $0.51 \mathrm{meV}$ (случай с закрытыми концами). Аналогичный эффект появления запрещенной зоны величиной $0.2 \mathrm{eV}$ был обнаружен для 3D-сетки, образованной УНТ $(5,5)$, встроенной в графен с обеих сторон листа [44]. В работе [45] методами $a b$ initio проводится исследование транспортных свойств колонного графена, построенного на базе нанотрубок armchair $(4,4)$ и zigzag $(8,0)$. Установлено, что для колонного графена на основе металлических УНТ $(4,4)$ проводимость практически не зависит от длины трубки, но сильно меняется в зависимости от структуры контакта графен-УНТ, в то время как для колонного графена на основе полупроводниковых УНТ $(8,0)$ проводимость, главным образом, определяется длиной трубки и не зависит от структуры контакта графен-УНТ. В работах [46-48] на примере атомистических моделей колонного графена, образованного УНТ $(6,6)$, показано, что бесшовные 3D-структуры графен-УНТ демонстрируют превосходные теплопроводящие свойства, причем тепловой поток в таких структурах определяется минимальным расстоянием между трубками и их длиной [47].

В последние годы одним из перспективных направлений развития исследований в области графен-УНТ соединений является получение композитных материалов с улучшенными эмиссионными характеристиками для последующего применения в качестве наноэмиттеров [49-54]. Одним из последних достижений в этой области стало экспериментальное получение методом электрофоретического осаждения гибридных пленок ОУНТ/графен, демонстрирующих максимальный эмиссионный ток величиной $80 \mathrm{~mA} \mathrm{c}$ соответствующей плотностью тока $160 \mathrm{~mA} / \mathrm{cm}^{2}$ при напряженности электрического поля $9.6 \mathrm{~V} / \mu \mathrm{m}$ [54]. В то же время в этой и других подобных работах не рассматривается поведение гибридных структур графен/УНТ в сильных электрических полях напряженностью $10^{9}-10^{10} \mathrm{~V} / \mathrm{m}$, которая характерна для эмиссионных центров автокатодов. Понимание этого вопроса крайне важно для успешного применения графен-нанотрубных пленок в качестве источника электронов вакуумных электронных устройств.

Данная работа посвящена in silico исследованию влияния сильных электрических полей с напряженностью $10^{7}-10^{8} \mathrm{~V} / \mathrm{cm}$ и более на атомное и электронное строение квази-1D-структур на основе хиральной нанотрубки $(6,5)$ и графеновых структур, ковалентно соединенных с трубкой. Выбор моделируемого объекта обусловлен тем, что такие ковалентно соединенные графен-УНТ структуры синтезируются в реальном экс- перименте, в частности с помощью технологии лазерной наносварки [55].

\section{2. Математическое моделирование: подходы и методы}

В первую очередь были построены атомистические модели графен/нанотрубных квази-1D-структур. Построение проводилось с помощью метода функционала плотности в приближении сильной связи с самосогласованием заряда (метод SCC DFTB, не поляризованный по спину), реализованного в программном пакете DFTB+ [56]. Равновесная конфигурация суперъячеек тонких пленок достигалась минимизацией полной энергии при варьировании всех координат всех атомов суперьячейки и длины ее вектора трансляции. При этом для разбиения первой зоны Бриллюэна применялся подход Монкхорст-Пака [57], и исследования проводились на сетке $1 \times 4 \times 1$ (структура периодическая в направлении оси $Y$ ). Электронная заселенность рассматривалась при температуре $300 \mathrm{~K}$ и определялась функцией Ферми-Дирака

$$
f(\varepsilon)=\left[1+\exp \left(\varepsilon / k_{B} T\right)\right]^{-1} .
$$

Для учета возможного ван-дер-ваальсового взаимодействия (притяжения/отталкивания) отдельных конструктивных элементов квази-1D-структур в рамках метода SCC DFTB был применен универсальный потенциал силового поля (UFF) [58]. Для выявления закономерностей электронного строения рассчитывалась плотность электронных состояний (DOS) и энергия Ферми. Для исследования электропроводности квази-1D-структур применяется аппарат неравновесных функций Грина-Келдыша и формализм Ландауэра-Буттикера [59], который позволяет определить величину электропроводности $G$ на основе функции пропускания электронов $T(E)$ :

$$
G=\frac{2 e^{2}}{h} \int_{-\infty}^{\infty} T(E) F_{T}\left(E-E_{F}\right) d E
$$

где $e-$ заряд электрона, $h$ - постоянная Планка. Величина $e^{2} / h-$ квант проводимости. Значение кванта проводимости удваивается для учета спина электронов. Функция теплового уширения $F_{T}(E)$ рассчитывается по формуле:

$$
F_{T}(E)=\frac{1}{4 k_{B} T} \operatorname{sech}\left(\frac{E}{2 k_{B} T}\right),
$$

где $k_{B}-$ постоянная Больцмана, $T-$ температура. Функция пропускания электронов $T(E)$, как известно, характеризует квантово-механическую прозрачность проводящего канала (участка структуры, заключенного между двумя контактами) в зависимости от энергии движущегося по нему электрона. Суперьячейка структуры выступает в качестве канала, а в качестве 

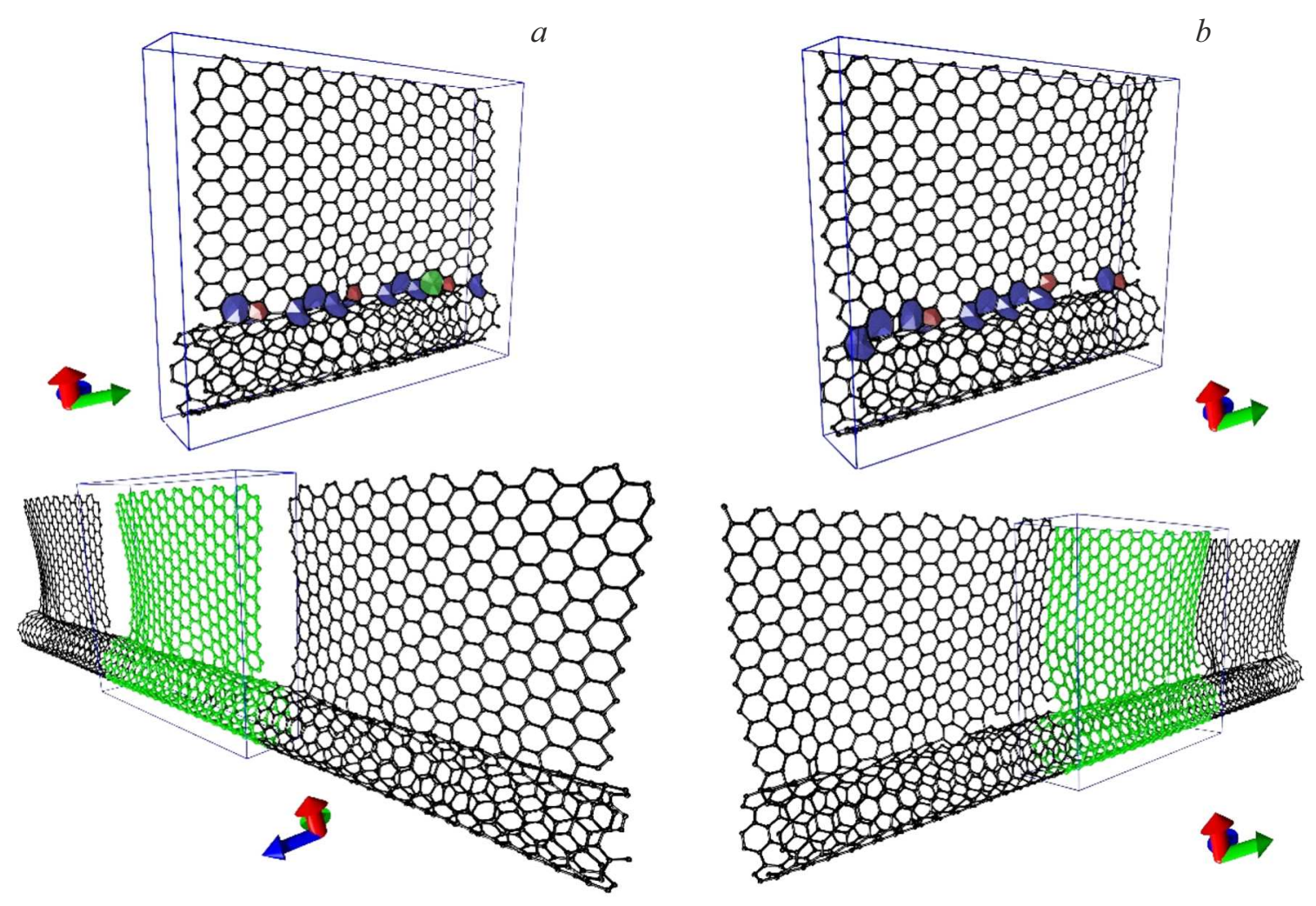

Рис. 1. Атомистические модели квази-1D-структуры с чешуйчатым графеном: (a) Q1D-1; (b) Q1D-2.

электродов - полубесконечные структуры, получаемые транслированием суперъячеек исследуемой структуры в двух противоположных направлениях. Используемый формализм предусматривает описание закономерностей когерентного транспорта электронов, когда при движении электрона через канал учитываются только упругие столкновения с атомами кристаллической решетки. Энергия рассчитывалась также с помощью метода SCC DFTB. Для выявления поведения структуры в электрическом поле применялась квантовая молекулярная динамика Борна-Оппенгеймера на основе метода SCC DFTB с применением расширенного лагранжиана [60]. Этот метод появился сравнительно недавно и является альтернативным подходом для квантового молекулярнодинамического моделирования. Он основан на расширенной лагранжевой формулировке молекулярной динамики Борна-Оппегеймера [61] и позволяет избежать недостатки предыдущих модификаций молекулярной динамики, сочетая в себе некоторые из лучших особенностей подхода Кара-Парринелло и Борна-Оппенгеймера. Расчеты проводились с временным шагом $0.5 \mathrm{fs}$.

\section{3. Атомистические модели}

Для построения атомистических моделей графен/ нанотрубных квази-1D-структур были выбраны хиральная нанотрубка $(6,5)$ и графеновые фрагменты. Трубка $(6,5)$ выбрана по той причине, что это одна из часто синтезируемых одностенных трубок субнанометрового диаметра $0.75 \mathrm{~nm}$. Длина вектора трансляции $L_{y}=4.064 \mathrm{~nm}$. На основе этой трубки были построены две модели. Одна из моделей (Q1D-1), содержащая 722 атома, представлена на рис. 1, $a$. Графеновая чешуйка содержит 358 атомов, трубка - 364 атома. Исходная длина вектора трансляции квази-1D-структуры Q1D-1 соответствовала рассчитанному периоду индивидуальной трубки $(6,5)$. Наименьшее расстояние между атомами графена и трубки составляло $\sim 1.65 \AA$. В результате оптимизации трубка и графен образовали ковалентные связи. Количество атомов в суперьячейке осталось прежним. Длины связей изменились, как и длина вектора трансляции суперъячейки. В области стыка находятся негексагональные элементы, как можно видеть на рис. 1, $a$ (в верхней части рисунка): пяти-/семии восьмиугольники. Пятиугольники отмечены красным, семиугольники - синим, восьмиугольник - зеленым. Сразу можно отметить, что негексагональные элементы распределены неравномерно вдоль атомного каркаса нанотрубки, что обуславливается хиральной структурой трубки и, в связи с этим, невозможностью появления одинаковых ковалентных связей по всей длине графена. Поэтому вблизи одного конца трубки, в области стыка графен-нанотрубка, обнаружилась сразу целая группа семи-/восьми-/пятиугольников, а вблизи другого конца трубки восьмиугольников вообще нет, и количество семи-/пятиугольников значительно меньше. Пятиуголь- 
ники отмечены красным цветом, семиугольники - синим, восьмиугольник - зеленым. На том же рис. 1, $a$ (нижняя часть рисунка) представлен фрагмент бесконечной квази-1D-структуры с представлением периодического ящика (периодический ящик отмечен синим цветом, а также атомы суперъячейки выделены зеленым цветом). На этом рисунке представлен такой ракурс квази-1D-структуры, чтобы было лучше увидеть топологию. Действительно, хорошо видно, что графеновые чешуйки как бы отталкиваются друг от друга, образуя свободное пространство между ними. Расстояние между атомами соседних графеновых чешуек составляет $\sim 5.6 \AA$ и более. Из-за отталкивания графеновых чешуек друг от друга длина структуры увеличилась, и вектор трансляции после оптимизации имеет длину $4.09 \mathrm{~nm}$. Таким образом, топология первой модели квази-1D-структуры (Q1D-1) характеризуется в некоторых областях отсутствием ковалентных связей между графеновой лентой и нанотрубкой. Все топологические и метрические характеристики представлены в табл. 1.

Другая модель (Q1D-2), построенная на основе той же нанотрубки $(6,5)$, отличается тем, что графен представлен сплошной 1D-нанолентой, присоединенной ковалентно к нанотрубке. Изначальная атомистическая модель суперъячейки имела длину вектора трансляции, равную $L_{y}=4.064 \mathrm{~nm}$, наименьшее расстояние между атомами графена и трубки также составляло $\sim 1.65 \AA$. Количество атомов трубки $(6,5)$ осталось прежним, но количество атомов графеновой чешуйки было увеличено до 380. То есть, длина графеновой чешуйки была максимально приближена к длине исходной индивидуальной трубки для обеспечения образования ковалентных связей не только между графеном и трубкой, но и между соседними графеновыми чешуйками. Из-за хиральности трубки $(6,5)$ невозможно обеспечить однородную связь графен-трубка и планарную топологию графеновой наноленты вдоль трубки, но можно получить гофрированный графен, соединенный с трубкой. Это и было достигнуто. На рис. $1, b$ представлена полученная в результате оптимизации атомистическая структура модели квази-1D-структуры с протяженным графеновым листом, ковалентно присоединенным к нанотрубке. На этом же рисунке показано появление негексагональных элементов в области стыка графен-трубка. Для этой модели характерно появление пяти-/семиугольников, но без восьмиугольников. Также характерно несимметричное распределение негексагональных элементов в области стыка графена с нанотрубкой. На рис. 1, $b$ представлен фрагмент протяженной квази-1D-структуры с указанием суперъячейки, отмеченной зеленым цветом. Можно увидеть, что протяженный фрагмент графеновой ленты нерегулярно связан с нанотрубкой, то есть существуют области без ковалентных связей графен-нанотрубка. Как упоминалось выше, это вполне ожидаемо из-за хиральной структуры атомного остова трубки. Топологические и метрические характеристики Q1D-2 модели представлены в табл. 1. Следует отметить,
Таблица 1. Геометрические и энергетические параметры суперъячейки графен/нанотрубных квази-1D-структур

\begin{tabular}{l|c|c}
\hline \multicolumn{1}{c|}{ Параметры } & Q1D-1 & Q1D-2 \\
\hline Количество пятиугольников & 5 & 6 \\
Количество семиугольников & 11 & 8 \\
Количество восьмиугольников & 2 & 0 \\
Длина ковалентной связи между & 1.56 & 1.57 \\
графеном и УНT, $\AA$ & & \\
Длина структуры вдоль оси $X, \AA$ & 33.00 & 33.12 \\
Вектор трансляции $L_{y}, \AA$ & 40.90 & 40.64 \\
Энергия формирования, eV/atom & -47.14 & -47.18
\end{tabular}

что в результате оптимизации длина вектора трансляции не изменилась, но графеновый лист потерял один атом при образовании связей с трубкой, что привело к формированию пятиугольника в центральной части. Таким образом, в суперъячейке стало 743 атома вместо 744-х. Это позволило получить структуру графен/нанотрубка, наиболее приближенную по своей топологии к синтезируемым.

Из данных табл. 1 следует, что длины связи в области стыка графена и УНТ соответствуют состоянию $s p^{3}$-гибридизации, поскольку атомы этой области формируют 4 ковалентных связи. Также видно, что квази-1D-структура с протяженным листом графена, в большей части присоединенным к протяженной трубке $(6,5)$, является наиболее энергетически выгодным вариантом. Это можно объяснить тем фактом, что графеновые чешуйки (рис. $1, a$ ) отталкиваются друг от друга, что создает определенное напряжение в атомной структуре. При этом, несмотря на гофрированность графена модели Q1D-2, длины связей находятся в пределах типичных для графена значений и составляют 1.40-1.42 А. В целом, обе модели энергетически выгодны с позиции их формирования, так как энергия формирования, рассчитываемая как разность энергий конечной структуры и начальных отдельных фрагментов, является отрицательной.

\section{4. Электронные и электрофизические характеристики}

Для построенных моделей квази-1D-структур были рассчитаны плотности электронных состояний, энергия Ферми, а также исследованы транспортные электронные свойства, для чего рассчитывалась функция пропускания $T(E)$, затем электропроводность $G$ и электрическое сопротивление. На рис. 2, $a$ представлены графики плотности электронных состояний для обеих моделей квази-1D-структур, а также отмечены величины энергии Ферми, заметно отличающиеся для двух моделей. На рис. 2, $b$ показаны графики функций пропускания. Электронные и электрофизические параметры исследуемых квази-1D-структур представлены в табл. 2. Энергия 


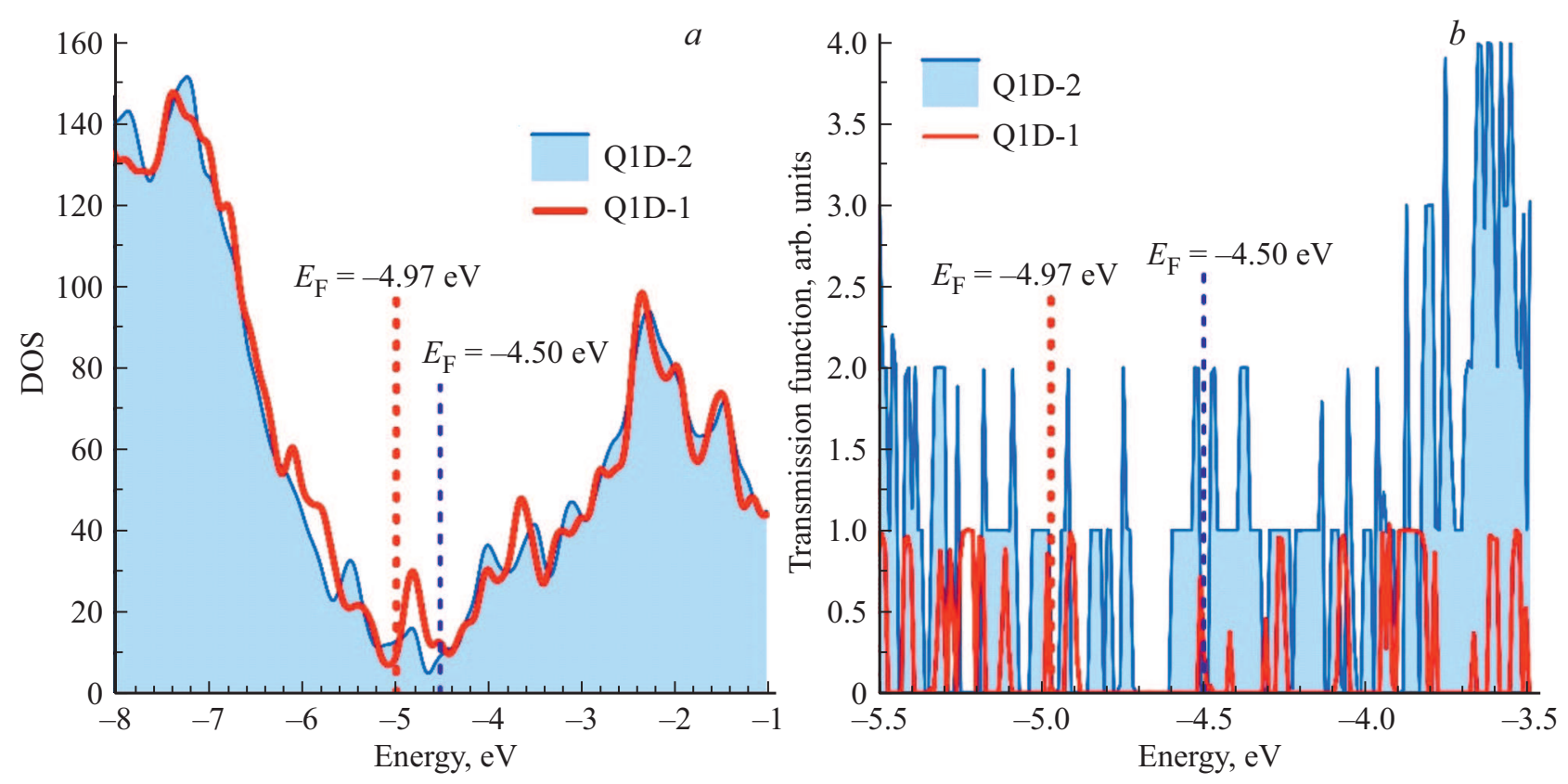

Рис. 2. Графики плотности электронных состояний $(a)$ и функции пропускания $(b)$ графен/нанотрубных квази-1D-структур.

Ферми для модели Q1D-2 с протяженным листом графеновой ленты близка к значению для чистого монослоя графена $(4.61 \mathrm{eV})$, энергетическая щель в зонной структуре практически отсутствует. Для варианта с графеновыми чешуйками Q1D-1 энергия Ферми больше отвечает значению для индивидуальных нанотрубок, но энергетическая щель также незначительна. Графики плотности электронных состояний в обоих случаях даже не отображают энергетическую щель. Таким образом, обнаружен очень интересный физический факт, требующий обсуждения и понимания: при наличии энергетической щели у исходной индивидуальной хиральной трубки $(6,5)$ и у графеновой ленты типа „кресло“ гибридные квази-1D-структуры становятся проводящими.

Для объяснения этого эффекта были исследованы электронные характеристики индивидуальной протяженной хиральной трубки $(6,5)$ и графеновой протяженной наноленты типа „кресло“, фрагменты которой применялись для формирования графен/нанотрубных квази-1D-структур. Как видно из рис. 1, в качестве протяженной графеновой наноленты выступала нанолента кресельного типа AGNR, которая и контактировала с трубкой. Для обеих моделей была выбрана одна нанолента, только для модели Q1D-1 была взята чешуйка, т. е. часть ленты, а для второй модели Q1D-2 - протяженная нанолента. Энергетическая щель зонной структуры индивидуальной трубки $(6,5)$ составляет $0.9 \mathrm{eV}$, энергия Ферми составляет $-4.57 \mathrm{eV}$. У графеновой наноленты энергетическая щель равна $1 \mathrm{eV}$ (без пассивации краев) при значении для энергии Ферми $-5.72 \mathrm{eV}$. В настоящем исследовании не пассивировались края графеновых структур, поэтому и графеновая нанолента рассматривалась непассивированной. Как видно, обе исходные струк- туры являются полупроводниковыми, с одной стороны, и, с другой стороны, они обе характеризуются наличием только $s p^{2}$-гибридизованных электронных облаков у всех атомов. При формировании гибридной графен/нанотрубной структуры наблюдается $s p^{3}$-гибридизация электронных облаков атомов в области контакта и, как следствие, происходит частичное перетекание заряда с трубки на графеновую ленту.

Рассмотрим сначала вторую структуру Q1D-2, в которой графеновая лента протяженная, а не представлена чешуйками. В этом случае наблюдается частичное перетекание заряда с трубки на контактирующую графеновую наноленту. Это перетекание заряда довольно условное, поскольку есть ковалентная связь графен-нанотрубка, но в данном случае мы рассматриваем эти фрагменты отдельно, чтобы как-то охарактеризовать наблюдаемое перераспределение электронной плотности. Итак, величина заряда графена составляет $\sim-0.82 e$ ( $e-$ абсолютная величина заряда электрона), и эта величина характерная для суперьячейки, то есть, на протяжении всей структуры наблюдается перетекание заряда с трубки на графен. При этом все атомы, образующие ковалентные связи с графеном, имеют дефицит заряда $\sim 0.13-0.14 e$. Как известно, ранее Малликеном было предложено оценивать распределение электронной плотности по атомам структуры именно в единицах электронного заряда. Этот подход помогает понять, где именно находятся в структуре локальные области с избыточным электронным зарядом. Естественно, в таком подходе не имеется в виду, что половина электрона перешла с трубки на графен, а подразумевается перераспределение плотности электронного заряда, то есть расчет карты плотности вероятности для общего элек- 

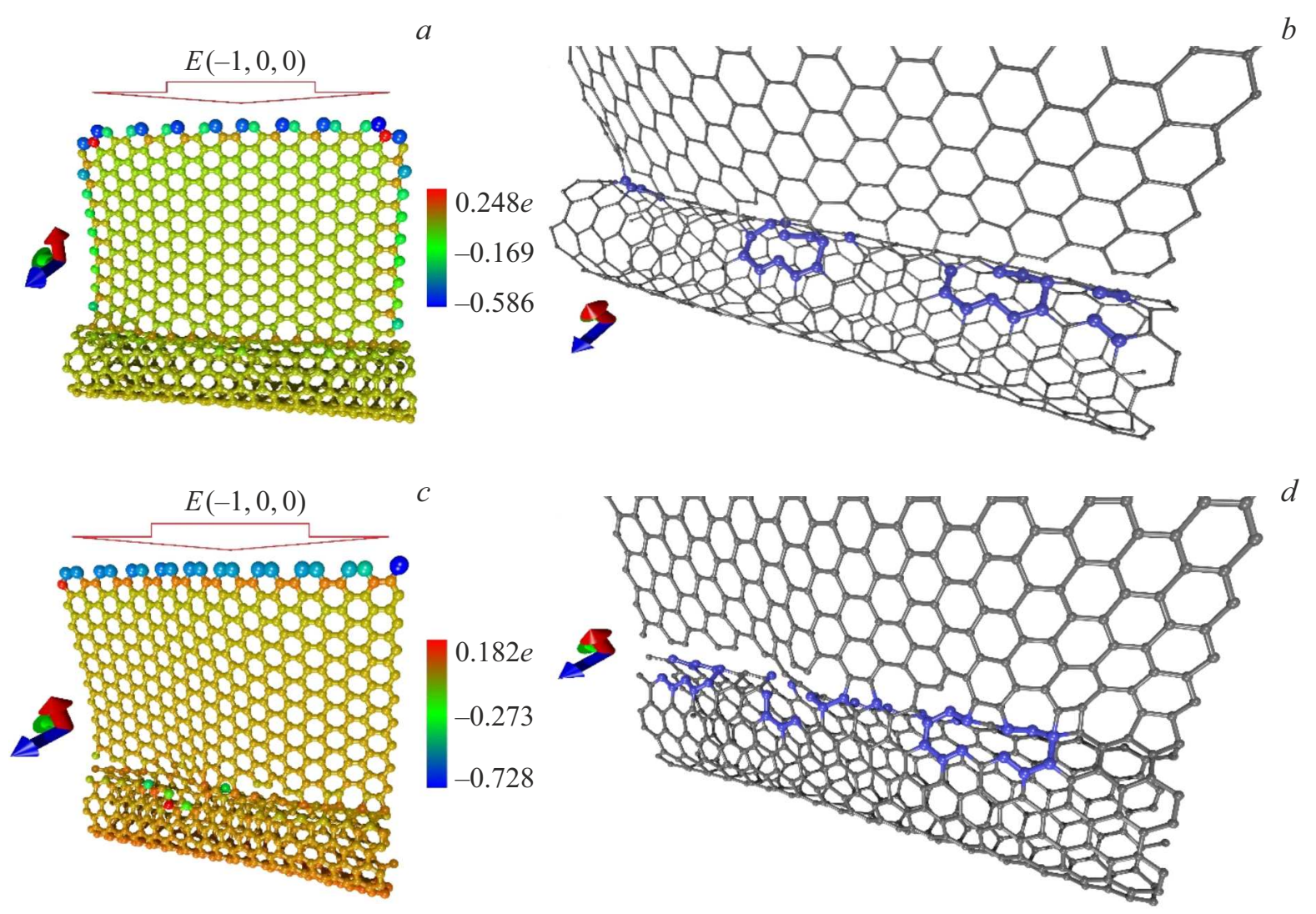

Рис. 3. Квази-1D-структуры в электрическом поле с напряженностью $1.5 \cdot 10^{8} \mathrm{~V} / \mathrm{cm}$ : распределение плотности электронного заряда и фрагмент разрушающегося остова для модели Q1D-1 $(a, b)$ и для модели Q1D-2 $(c, d)$.

тронного облака графен/нанотрубной структуры. Возвращаясь к вышеизложенному, замечаем, что графеновая лента, ковалентно присоединенная к нанотрубке, имеет избыточный электронный заряд, что заметно меняет электронные свойства всей квази-1D-структуры. В связи с этим вблизи уровня Ферми с обеих сторон (рис. 2, $b$ ) плотность электронных состояний отлична от нуля, а график функции пропускания демонстрирует два пика интенсивности на уровне „2“. Величина функции пропускания представлена в относительных единицах квантах проводимости $e^{2} / h$. Таким образом, электрическое сопротивление полученной графен/нанотрубной квази-1D-структуры является достаточно небольшим (табл. 2), всего лишь $\sim 12.8 \mathrm{k} \Omega$, что близко к сопротивлению идеальных трубок типа „кресло“ $6.4 \mathrm{k} \Omega$. Другая структура Q1D-1 характеризуется несколько большим сопротивлением, уровень Ферми смещен в область меньших значений (табл. 2). Профиль функции пропускания совершенно отличен от профиля для структуры Q1D-2. Рядом с уровнем Ферми есть пик интенсивности, но его величина равна единице и, как видно из графика $T(E)$, интенсивность функции пропускания не достигает „2“ на всем промежутке энергий. Такой результат для структуры Q1D-1 вполне ожидаем. Так, например, графеновые чешуйки не могут взять на себя большой заряд, а только $\sim-0.58 e$ (на суперъячейку).
Таблица 2. Электронные и электрофизические характеристики графен/нанотрубных квази-1D-структур

\begin{tabular}{l|c|c}
\hline \multicolumn{1}{c|}{ Параметры } & Q1D-1 & Q1D-2 \\
\hline Энергия Ферми, eV & -4.97 & -4.50 \\
Энергетическая щель, eV & 0.08 & 0.04 \\
Электропроводность, $\mu \mathrm{S}$ & 13.064 & 78.400 \\
Сопротивление, kOhm & 76.545 & 12.755
\end{tabular}

Подытоживая полученную информацию об электронном строении, можно заключить, что формирование нового комплекса из графеновой наноленты/чешуек и нанотрубки обеспечивает этому комплексу совершенно новое электронное строение, не повторяющее ни электронное строение нанотрубки, ни графеновой наноленты.

\section{5. Поведение в сильных электрических полях}

Далее было исследовано поведение квази-1D-структур в сильных электрических полях, которые необходимы для автоэлектронной эмиссии. Прежде всего необходимо установить критические поля, которые будут разрушать структуру. В настоящей работе квази-1D-структура рас- 


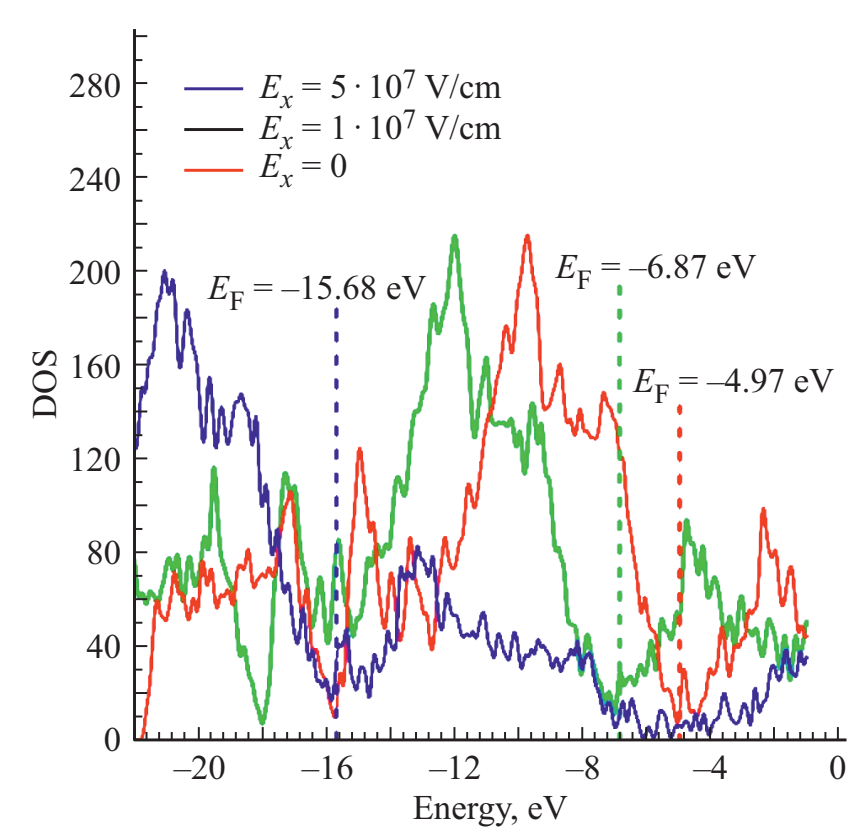

Pис. 4. Плотность электронных состояний квази-1D-структуры Q1D-1 при различных значениях напряженности внешнего электрического поля.

полагается таким образом, что представляет собой лезвийный автоэмиссионный катод. Направление силовых линий показано на рис. 3. Внешнее поле приводит к перераспределению плотности электронного заряда у структуры. Естественно, максимальный избыточный заряд наблюдается у краевых атомов графена (они представлены синим цветом), то есть поле как бы „стягивает“ электронный заряд со всей структуры на край. Исследовано поведение в полях с напряженностью от $1 \cdot 10^{7}$ до $2 \cdot 10^{8} \mathrm{~V} / \mathrm{cm}$. Обнаружено, что частичное разрушение начинается при напряженности поля $1.5 \cdot 10^{8} \mathrm{~V} / \mathrm{cm}$, что и отображено на рис. 3. На рис. $3, a, b$ представлены карта распределения плотности электронного заряда и атомная сетка первой модели Q1D-1 с графеновой чешуйкой. Частичные разрушения происходят уже в течение первой пикосекунды с момента включения внешнего поля. На рис. $3, b$ видно, что чешуйка практически полностью отрывается полем от нанотрубки, и сама трубка в месте отрыва терпит локальные разрывы ковалентных связей (эти области отмечены синим цветом). Аналогичная ситуация наблюдается и для другой модели Q1D-2 (рис. $3, c, d$ ). При том же значении напряженности поля у этой структуры графен отрывается только частично, однако отрыв части графена приводит к значительному разрыву остова трубки. Таким образом, поле не только тянет графен, но и стремится его разорвать по шву контакта трубка-графен. При увеличении напряженности поля до $\sim 2 \cdot 10^{8} \mathrm{~V} / \mathrm{cm}$ происходит полный отрыв графена.

При меньших значениях напряженности $\sim 1 \cdot 10^{8} \mathrm{~V} / \mathrm{cm}$ также наблюдается частичное разрушение после первой пикосекунды молекулярно-динамического моделирования. Однако, частичные разрывы в области контакта графен-трубка далее сохраняются, и более структура не разрушается. Изменения профиля DOS для обеих структур во внешнем поле идентичны. Уровень Ферми смещается в область отрицательных значений. На рис. 4 показаны графики DOS для модели Q1D-1 для трех различных случаев величины напряженности. Видно смещение профиля DOS вместе с уровнем Ферми. Аналогичная картина наблюдается и для структуры Q1D-2.

\section{6. Заключение}

Получены новые знания о влиянии сильных электрических полей на атомную структуру и электронное строение перспективных для автоэмиссионной электроники графен/нанотрубных квази-1D-структур. Рассмотрены два типа топологических моделей композита: 1) на основе хиральной нанотрубки $(6,5)$ и графеновых чешуек; 2) на основе хиральной нанотрубки $(6,5)$ и графеновой наноленты типа „кресло“. Показано, что для обеих моделей характерно несимметричное распределение негексагональных элементов в области контакта графен-нанотрубка, обусловленное хиральной структурой трубки. Обнаружено, что несмотря на полупроводниковый характер проводимости трубки $(6,5)$ и кресельной графеновой наноленты, полученные из них гибридные квази-1D-структуры становятся проводящими за счет частичного перетекания заряда с нанотрубки на графеновую наноленту, вызванного $s p^{3}$-гибридизацией электронных облаков атомов в области контакта графен-нанотрубка. Впервые установлены критические значения напряженности электрического поля, приводящие к разрушению рассмотренных квази-1D-структур. Выявлено, что частичное разрушение структуры в виде отрыва графеновых фрагментов от нанотрубки и локального разрыва связей между атомами нанотрубки в квази-1D-структурах начинается при напряженности поля $1.5 \cdot 10^{8} \mathrm{~V} / \mathrm{cm}$. Показано, что для обеих моделей графен/нанотрубных квази-1D-структур во внешнем поле наблюдается смещение уровня Ферми в область отрицательных значений энергий. Полученные новые знания важны для оценки автоэмиссионных свойств подобных квази-1D-структур, которые могут найти применение в качестве лезвийного автоэмиссионного катода.

\section{Финансирование работы}

Работа выполнена при поддержке гранта Российского научного фонда (проект № 21-19-00226).

\section{Конфликт интересов}

Авторы заявляют, что у них нет конфликта интересов. 


\section{Список литературы}

[1] A.K. Geim, K.S. Novoselov. Nature Mater. 6, 183 (2007).

[2] V. Georgakilas, J.A. Perman, J. Tucek, R. Zboril. Chem. Rev. 115, 11, 4744 (2015).

[3] M.F.L. De Volder, S.H. Tawfick, R.H. Baughman, A.J. Hart. Science 339, 6119, 535 (2013).

[4] V.T. Dang, D.C. Nguyen, T.T. Cao, P.H. Le, D.L. Tran, N.M. Phan, V.C. Nguyen. Adv. Nat. Sci.: Nanosci. Nanotechnol. 7, 3, 033002 (2016).

[5] Y. Li, Q. Ai, L. Mao, J. Guo, T. Gong, Y. Lin, G. Wu, W. Huang, X. Zhang. Sci. Rep. 11, 21006 (2021).

[6] R. Ghosh, T. Maruyama, H. Kondo, K. Kimoto, T. Nagai, S. Iijima. Chem. Commun. 51, 43, 8974 (2015).

[7] E. Shi, H. Li, L. Yang, J. Hou, Y. Li, L. Li, A. Cao, Y. Fang. Adv. Mater. 27, 4, 682 (2015)

[8] Л.А. Чернозатонский, П.Б. Сорокин, А.А. Артюх. Успехи химии 83, 3, 251 (2014).

[9] A.L. Gorkina, A.P. Tsapenko, E.P. Gilshteyn, T.S. Koltsova, T.V. Larionova, A. Talyzin, A.S. Anisimov, I.V. Anoshkin, E.I. Kauppinen, O.V. Tolochko, A.G. Nasibulin. Carbon 100, 501 (2016).

[10] W. Du, Z. Ahmed, Q. Wang, C. Yu, Z. Feng, G. Li, M. Zhang, C. Zhou, R. Senegor, C.Y. Yang. 2D Mater. 6, 4, 042005 (2019).

[11] G.K. Dimitrakakis, E. Tylianakis, G.E. Froudakis. Nano Lett. 8, 10, 3166 (2008).

[12] W. Wang, M. Ozkan, C.S. Ozkan. J. Mater. Chem. A 4, 9, 3356 (2016).

[13] M.Q. Zhao, X.F. Liu, Q. Zhang, G.L. Tian, J.Q. Huang, W. Zhu, F. Wei. ACS Nano 6, 12, 10759 (2012).

[14] Y. Zhu, L. Li, C. Zhang, G. Casillas, Z. Sun, Z. Yan, G. Ruan, Z. Peng, A.R.O. Raji, C. Kittrell, R.H. Hauge, J.M. Tour. Nature Commun. 3, 1225 (2012).

[15] F. Du, D. Yu, L. Dai, S. Ganguli, V. Varshney, A.K. Roy. Chem. Mater. 23, 21, 4810 (2011).

[16] A. Hassani, M.T.H. Mosavian, A. Ahmadpour, N. Farhadian. J. Chem. Phys. 142, 23, 234704 (2015).

[17] I.N. Kholmanov, C.W. Magnuson, R. Piner, J.Y. Kim, A.E. Aliev, C. Tan, T.Y. Kim, A.A. Zakhidov, G. Sberveglieri, R.H. Baughman, R.S. Ruoff. Adv. Mater. 27, 19, 3053 (2015).

[18] F. Tristán-López, A. Morelos-Gómez, S.M. Vega-Díaz, M.L. García-Betancourt, N. Perea-López, A.L. Elías, H. Muramatsu, R. Cruz-Silva, S. Tsuruoka, Y.A. Kim, T. Hayahsi, K. Kaneko, M. Endo, M. Terrones. ACS Nano 7, 12, 10788 (2013)

[19] S.H. Kim, W. Song, M.W. Jung, M.A. Kang, K. Kim, S.J. Chang, S.S. Lee, J. Lim, J. Hwang, S. Myung, K.S. An. Adv. Mater. 26, 25, 4247 (2014).

[20] X. Gan, R. Lv, J. Bai, Z. Zhang, J. Wei, Z.H. Huang, H. Zhu, F. Kang, M. Terrones. 2D Mater. 2, 3, 034003 (2015).

[21] Z. Yan, Z. Peng, G. Casillas, J. Lin, C. Xiang, H. Zhou, Y. Yang, G. Ruan, A.R. Raji, E.L. Samuel, R.H. Hauge, M.J. Yacaman, J.M. Tour. ACS Nano 8, 5, 5061 (2014).

[22] Z. Chen, T. Lv, Y. Yao, H. Li, N. Li, Y. Yang, K. Liu, G. Qian, X. Wang, T. Chen. J. Mater. Chem. A 7, 43, 24792 (2019).

[23] X. Yang, D. Yu, B. Cao, A.C. To. ACS Appl. Mater. Interfaces 9, 1, 29 (2017).

[24] K. Duan, L. Li, Y. Hu, X. Wang. Sci. Rep. 7, 14012 (2017).
[25] A. Pedrielli, S. Taioli, G. Garberoglio, N.M. Pugno. Micropor. Mesopor. Mater. 257, 222 (2018).

[26] J. Chen, J.H. Walther, P. Koumoutsakos. Nanotechnology 27, 465705 (2016).

[27] Y. Liu, Y. Liu, S. Qin, Y. Xu, R. Zhang, F. Wang. Nano Res. 10, 6, 1880 (2016).

[28] B. Cai, H. Yin, T. Huo, J. Ma, Z. Di, M. Li, N. Hu, Z. Yang, Y. Zhang, Y. Su. J. Mater. Chem. C 8, 10, 3386 (2020).

[29] B. Liu, M. Alamri, M. Walsh, J.L. Doolin, C.L. Berrie, J.Z. Wu. ACS Appl. Mater. Interfaces 12, 47, 53115 (2020).

[30] H. Kim, J. Kim, H.S. Jeong, H. Kim, H. Lee, J.M. Ha, S.M. Choi, T.H. Kim, Y.C. Nah, T.J. Shin, J. Bang, S.K. Satijag, J. Koo. Chem. Commun. 54, 41, 5229 (2018).

[31] Q. Cheng, J. Tang, J. Ma, H. Zhang, N. Shinyaa, L.-C. Qinc. Phys. Chem. Chem. Phys. 13, 39, 17615 (2011).

[32] R.T. Lv, E. Cruz-Silva, M. Terrones. ACS Nano 2014, 8, 5, 4061.

[33] X.L. Li, J.W. Sha, S.K. Lee, Y.L. Li, Y.S. Ji, Y.J. Zhao, J.M. Tour. ACS Nano 10, 8, 7307 (2016).

[34] L. Cai, X. Xue, M. Liu, H. Li, X. Zhou, G. Yu. APL Materials 9, 4, 041110 (2021).

[35] Е.Ф. Шека, Л.А. Чернозатонский, А.А. Артюх. Письма в ЖЭТФ 89, 7, 412 (2009).

[36] L.A. Chernozatonskii, P.B. Sorokin. ECS Trans. 19, 13, 35 (2009).

[37] E.F. Sheka, L.A. Chernozatonskii. J. Comput. Theor. Nanosci. 7, 9, 1814 (2010).

[38] A.A. Artyukh, L.A. Chernozatonskii, P.B. Sorokin. Phys. Status Solidi B 247, 11-12, 2927 (2010).

[39] V.V. Ivanovskaya, A. Zobelli, P. Wagner, M.I. Heggie, P.R. Briddon, M.J. Rayson, C.P. Ewels. Phys. Rev. Lett. 107, 6, 065502 (2011).

[40] M.A. Akhukov, S. Yuan, A. Fasolino, M.I. Katsnelson. New J. Phys. 14, 123012 (2012).

[41] O.E. Glukhova, I.S. Nefedov, A.S. Shalin, M.M. Slepchenkov. Beilstein J. Nanotechnol. 9, 1321 (2018).

[42] J. Gonga, P. Yang. RSC Adv. 4, 38, 19622 (2014).

[43] T. Matsumoto, S. Saito. J. Phys. Soc. Jpn. 71, 2765 (2002).

[44] Y. Mao, J. Zhong. New J. Phys. 11, 093002 (2009).

[45] F.D. Novaes, R. Rurali, P. Ordejón. ACS Nano 4, 12, 7596 (2010).

[46] J. Chen, J.H. Walther, P. Koumoutsakos Adv. Funct. Mater. 25, 7539 (2015)

[47] V. Varshney, S.S. Patnaik, A.K. Roy, G. Froudakis, B.L. Farmer. ACS Nano 4, 2, 1153 (2010).

[48] Z. Zhang, A. Kutana, A. Roy, B.I. Yakobson. J. Phys. Chem. C 121, 2, 1257 (2017).

[49] D.D. Nguyen, R.N. Tiwari, Y. Matsuoka, G. Hashimoto, E. Rokuta, Y. Chen, Y.L. Chueh, M. Yoshimura. ACS Appl. Mater. Interfaces 6, 12, 9071 (2014).

[50] M. Song, P. Xu, Y. Song, X. Wang, Z. Li, X. Shang, H. Wu, P. Zhao, M. Wang. AIP Adv. 5, 9, 097130 (2015).

[51] S. Riyajuddin, S. Kumar, K. Soni, S.P. Gaur, D. Badhwar, K. Ghosh. Nanotechnology 30, 38, 385702 (2019).

[52] A.T.T. Koh, T. Chen, L. Pan, Z. Sun, D.H.C. Chua. J. Appl. Phys. 113, 17, 174909 (2013).

[53] L. Chen, H. He, H. Yu, Y. Cao, D. Lei, Q. Menggen, C. Wu, L. Hu. J. Alloys Compd. 610, 659 (2014).

[54] X. Hong, W. Shi, H. Zheng, D. Liang. Vacuum 169, 108917 (2019). 
[55] A.Y. Gerasimenko, A.V. Kuksin, Y.P. Shaman, E.P. Kitsyuk, Y.O. Fedorova, A.V. Sysa, A.A. Pavlov, O.E. Glukhova. Nanomaterials 11, 8, 1875 (2021).

[56] B. Hourahine, B. Aradi, V. Blum, F. Bonafé, A. Buccheri, C. Camacho, C. Cevallos, M.Y. Deshaye, T. Dumitrică, A. Dominguez, S. Ehlert, M. Elstner, T. van der Heide, J. Hermann, S. Irle, J.J. Kranz, C. Köhler, T. Kowalczyk, T. Kubař, I.S. Lee, V. Lutsker, R.J. Maurer, S.K. Min, I. Mitchell, C. Negre, T.A. Niehaus, A.M.N. Niklasson, A.J. Page, A. Pecchia, G. Penazzi, M.P. Persson, J. Řezáč, C.G. Sánchez, M. Sternberg, M. Stöhr, F. Stuckenberg, A. Tkatchenko, V.W.Z. Yu, T. Frauenheim. J. Chem. Phys. 152, 12, 124101 (2020).

[57] H.J. Monkhorst, J.D. Pack. Phys. Rev. B 13, 12, 5188 (1976).

[58] A.K. Rappe, C.J. Casewit, K.S. Colwell, W.A. Goddard III, W.M. Skiff. J. Am. Chem. Soc. 114, 25, 10024 (1992).

[59] S. Datta. Quantum Transport: Atom to Transistor. Cambridge University Press, N. Y. (2005). 432 p.

[60] B. Aradi, A.M.N. Niklasson, T. Frauenheim. J. Chem. Theory Comput. 11, 7, 3357 (2015).

[61] R. Car, M. Parrinello. Phys. Rev. Lett. 55, 22, 2471 (1985).

Редактор Ю.Э. Китаев 